\title{
EXTENDING THE OBSERVABLE ZENITH ANGLE OF FAST USING AN OFFSET FEED
}

\author{
LI GUODING \\ State Key Laboratory on Microwave and Digital Communication, The Department of Electronic \\ Engineering, Tsinghua University, Beijing 100084, China \\ R. NAN and B. PENG \\ Beijing Astronomical Observatory, National Astronomical Observatories, CAS, Beijing I00012, \\ China
}

\begin{abstract}
The five hundred meter aperture spherical radio telescope is will use an active spherical reflector. When the zenith scan angle is changed, the illuminated part of the reflecting surface is made to fit a paraboloid of revolution in real time by active control. The maximum zenith scan angle $\psi_{\text {max }} \mid$ of FAST is $30^{\circ}$ under conditions of the geometry selected in order that the feed does not illuminate the ground. The result of this paper shows that the maximum zenith scan angle $\left|\psi_{\max }\right|$ can be extended to $69^{\circ}$ by offsetting the feed.
\end{abstract}

Keywords: radio telescope, maximum zenith scan angle, offset-fed

\section{Introduction}

The Five hundred meter Aperture Spherical radio Telescope (FAST) is a giant karst-based radio telescope (Qiu, 1998; Peng et al., 1996). It differs from the $305 \mathrm{~m}$ Arecibo telescope (Kildal et al., 1994) in that FAST will have an active spherical reflector. This active reflector consists of many elementary hexagonal segments having an open width of $L \mathrm{~m}$. When zenith scan angles are changed the illuminated part of the reflecting surface is made to fit a paraboloid of revolution in real time by active control. The radius $R$ of the spherical surface of FAST is $300 \mathrm{~m}$, and the opening $D$ of the spherical surface is $520 \mathrm{~m}$ diameter. The diameter $d$ of the aperture illuminated by the feed is $300 \mathrm{~m}$, the focal length of the paraboloid will be $F=0.467 R$. The maximum zenith scan angle $\left|\psi_{\max }\right|$ of the FAST is $30^{\circ}$ under conditions of the selected geometry to ensure that the feed pattern does not illuminate the ground. For angles $\left|\psi_{\max }\right|$ larger than $30^{\circ}$, there are the following two problems:

1. Part of the power radiated by the feed system will illuminate the ground. The spillover will reduce the gain of the antenna and increase the thermal noise produced by radiation from the ground.

2. The area of the illuminated aperture will reduce, which will influence the electrical performance of the antenna. 
The problems can be overcome by an offset feed for the active reflector to extend the angle $\left|\psi_{\max }\right|$. An offset-fed also influences the electric performance of the antenna. This paper investigates the dependence of the electric performance of the antenna on the zenith scan range of FAST when the angle $\left|\psi_{\max }\right|$ is extended by an offset-fed.

\section{Limit on Extended Zenith Scan Ranges of FAST by Offset Feed}

The maximum zenith scan angle $\left|\psi_{\max }\right|$ of FAST extended by an offset feed is limited by the following three factors.

1. The maximum adjustable distance $\delta_{\max }$ of the elementary spherical units along the radial direction. When the angle $\left|\psi_{\max }\right|$ is extended from $30^{\circ}$ to $30^{\circ}+\psi_{0}$ by an offset feed, the opening angle of the asymmetrically illuminated area is supposed to remain $60 \mathrm{deg}$, regardless of whether it can be realized by the same feed. The area would in fact be part of a new paraboloid with its apex at the intersection of the major optical axis and the spherical surface. The diameter of this (imaginary) paraboloid becomes d', where $d^{\prime}=2 R \sin \mid$ $\psi_{\max } \mid=2 R \sin \left(30^{\circ}+\psi_{0}\right) \geq 300 \mathrm{~m}$. So, the distance $\delta_{\max }$ must be increased and it increases quickly with increasing angle $\left|\psi_{\max }\right|$. For example, when the angle $\left|\psi_{\max }\right|$ of FAST is extended from $30^{\circ}$ to $45^{\circ}$ by an offset feed, the distance $\delta_{\max }$ increases from $0.7 \mathrm{~m}$ to about $6.5 \mathrm{~m}$. It is very difficult to realize such a large adjustment distance $\delta_{\max }$ through engineering, or the cost will be very expensive.

2. Paraboloid antenna focal ratio limit on the angle $\left|\psi_{\max }\right|$. When an offset feed is used, the focal length of the paraboloid is supposed to still be $F=0.467 R$, hence, the dependence of the parabolic antenna focal ratio $F / d^{\prime}$ on the angle $\left|\psi_{\max }\right|$ is $F / d^{\prime}=0.2335 / \sin \left|\psi_{\max }\right|$. When the angle $\left|\psi_{\max }\right| \geq 69^{\circ}$, the ratio $F / d^{\prime} \leq 0.25$ and the antenna changes from a paraboloid of long focal length to one of short focal length. When $F / d^{\prime}<0.25$ there are two bad electric current regions on the parabolic surface. The direction of one component of the current in one region is opposite to that of the current in the other region. The field radiated by them is of opposite phase at the maximum radiation direction of the antenna. So, it is clear there will be reduced performance of the antenna when the ratio $F / d^{\prime}<0.25$. Hence, the angle $\left|\psi_{\max }\right|$ of FAST can be at most extended by an offset feed to $69^{\circ}$.

3. Influence of offset feed on antenna gain. After implementation of an offset feed, the following two factors will influence the antenna gain. First, the area of the reflecting surface to be illuminated is supposed to still be a circle of $300 \mathrm{~m}$ diameter, but the effective aperture of the antenna is changed into an ellipse (dotted line), see Figure $1 \mathrm{~b}$. The antenna gain will reduce as the effective aperture decreases. Second, the subtended angle $2 \phi$ (see Figure 1a.) of the illuminated aperture at the phase center of the feed reduces rapidly with the 


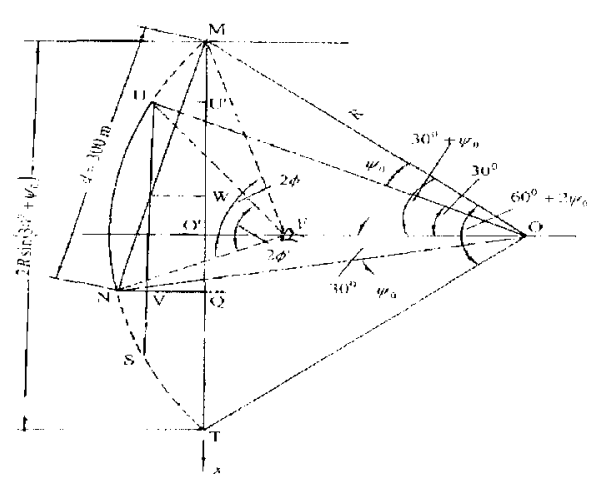

(a)

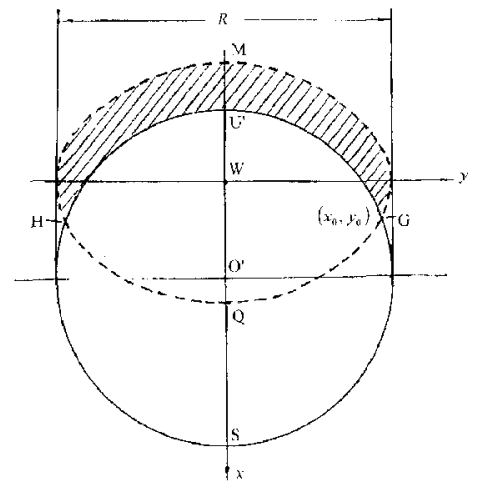

(b)

Figure 1 . The effective aperture of the antenna.

increase of the angle $\left|\psi_{\max }\right|$. For example, when the angle $\left|\psi_{\max }\right|$ of FAST is extended from $30^{\circ}$ to $45^{\circ}$ by an offset feed, the subtended angle $2 \phi$ decreases from $112.69^{\circ}$ to $107^{\circ}$. Hence, the spillover of the feed increases quickly and the antenna gain will be reduced. Now dependence of the antenna performances on the angle $\left|\psi_{\max }\right|$ will be calculated.

\section{Dependence of the Antenna Performance on the Maximum Zenith Scan} Angle

\subsection{DEPENDENCE OF THE ANTENNA GAIN ON THE ANGLE}

The distance $\left|\delta_{\max }\right|$ is limited to $0.70 \mathrm{~m}$ to reduce cost. The factors, except that of the feed spillover, which have influence on the antenna gain are supposed to be same. As shown in Figure $1 \mathrm{~b}$, when the angle $\left|\psi_{\max }\right| \leq 30^{\circ}$, the effective aperture of the antenna is the solid circle whose area is $S_{0}$. When the angle $\left|\psi_{\max }\right|>30^{\circ}$ and the distance $\delta_{\max }$ is not limited, the effective aperture of the antenna is the dotted ellipse shown in Figure $1 \mathrm{~b}$ whose area is $S_{e}$. As the angle $\left|\psi_{\max }\right|>30^{\circ}$ and the distance $\delta_{\max }$ is limited to $0.70 \mathrm{~m}$, the area of the effective aperture of the antenna is $S_{e}-S_{s}$ where $S_{s}$ is the area of the shadow shown in Figure 1b. The segments located in the shadow should be adjusted at random in order that the power reflected from the shadow is not received by the feed. The reduced value of the antenna gain is $\Delta G$ for the angle $\left|\psi_{\max }\right|$ that is extended by the offset feed, and

$$
\Delta G=\Delta G_{1}+\Delta G_{2}(\mathrm{~dB})
$$

where $\Delta G_{1}$ is the contribution of the decreased antenna effective aperture to the value $\Delta G$ and 


$$
\Delta G_{1}=\left\{\begin{array}{l}
101 g\left(\frac{S e}{S_{o}}\right)(\mathrm{dB}), \text { the } \delta_{\max } \text { is not limited } \\
101 g\left(\frac{S_{-}-S s}{S o}\right)(\mathrm{dB}), \text { the } \delta_{\max } \text { is limited to } 0.70 \mathrm{~m}
\end{array}\right.
$$

$\Delta G_{2}$ is the contribution of the incremental spillover of the feed to the value $\Delta G$. When the feed pattern is assumed to have the form $E(\phi)=\cos ^{n}(\phi / 2)$,

$$
\begin{aligned}
& \Delta G_{2}=\left\{101 g\left[1-\cos ^{(2 n+1)}(\phi / 2)\right]\right\}+0.4496, \quad \delta_{\max } \text { is not limited } \\
& \Delta G_{2}=101 g\left\{1-\frac{1}{2}\left[\cos ^{(2 n+1)}(\phi / 2)+\cos ^{(2 n+1)}\left(\phi^{\prime} / 2\right)\right]\right\}+0.4496(\mathrm{~dB}),
\end{aligned}
$$

Under the conditions of the geometry selected for FAST

$$
\begin{aligned}
& \phi=30^{\circ}+\frac{1}{2}\left\{\sin ^{-1}\left[\frac{0.533 \sin \left(60^{\circ}-\left|\psi_{\max }\right|\right)}{\sqrt{1.284-1.066 \cos \left(60^{\circ}-\left|\psi_{\max }\right|\right)}}\right]\right. \\
& \left.+\sin ^{-1}\left[\frac{0.533 \sin \left|\psi_{\max }\right|}{\sqrt{1.284-1.066 \cos \left|\psi_{\max }\right|}}\right]\right\} \\
& \phi^{\prime}=\frac{1}{2}\left\{116.3^{\circ}-\left|\psi_{\max }\right|+\sin ^{-1}\left[\frac{0.533 \sin \left(60^{\circ}-\left|\psi_{\max }\right|\right)}{\sqrt{1.284-1.066 \cos \left(60^{\circ}-\left|\psi_{\max }\right|\right)}}\right]\right\}
\end{aligned}
$$

According to formula (1), as $\mathbf{n}=8.2$ (the edge taper of the paraboloid is $-11 \mathrm{~dB}$ ) and the distance $\delta_{\max }$ is limited to $0.70 \mathrm{~m}$ and the zenith scan angle regions are respectively extended to $\pm 40^{\circ}, \pm 45^{\circ}, \pm 50^{\circ}$ and $\pm 55^{\circ}$ by the offset feed, the corresponding decrease in the antenna gain is $1.22 \mathrm{~dB}, 2.03 \mathrm{~dB}, 3.02 \mathrm{~dB}$ and $4.23 \mathrm{~dB}$, respectively.

\subsection{INFLUENCE OF THE ANGLE ON THE ANTENNA PATTERNS}

The angle $\left|\psi_{\max }\right|$ can be extended by the offset feed, which will also influence the antenna pattern. For the frequencies, f, of $1 \mathrm{GHz}, 2 \mathrm{GHz}$ and $5 \mathrm{GHz}$ and the angles $\left|\psi_{\max }\right|$ of $30^{\circ}, 55^{\circ}$ respectively, the parameters of the patterns of the E plane and $\mathrm{H}$ plane of the antenna are detailed in Table I.

\section{Conclusion}

From what is said above, the zenith scan region can be extended by an offset feed, but the antenna performance will be reduced whether the distance $\delta_{\max }$ is limited or not. If the distance $\delta_{\max }$ is limited, the antenna performance is reduced more quickly, so we have to accept a reduction in the antenna performance in exchange for extending the zenith scan region. The calculated results show that 
TABLE I

The parameters of the patterns

\begin{tabular}{|c|c|c|c|c|c|c|c|c|}
\hline \multirow{3}{*}{$\begin{array}{l}\left|\psi_{\max }\right| \\
f \\
(\mathrm{GHz})\end{array}$} & \multicolumn{4}{|c|}{$30^{\circ}$} & \multicolumn{4}{|c|}{$55^{\circ}$} \\
\hline & \multicolumn{2}{|c|}{$2 \beta$} & \multicolumn{2}{|c|}{$\xi(\mathrm{dB})$} & \multicolumn{2}{|c|}{$2 \beta$} & \multicolumn{2}{|c|}{$\xi(\mathrm{dB})$} \\
\hline & $\mathrm{E}$ & $\mathrm{H}$ & $\mathrm{E}$ & $\mathrm{H}$ & $\mathrm{E}$ & $\mathrm{H}$ & $\mathrm{E}$ & $\mathrm{H}$ \\
\hline 1.0 & $5.4^{\prime}$ & $5.4^{\prime}$ & -37.2 & -37.2 & $4.78^{\prime}$ & $6.56^{\circ}$ & -33.0 & -23.1 \\
\hline 2.0 & $2.7^{\prime}$ & $2.7^{\prime}$ & -37.2 & -37.2 & 2.39' & $3.28^{\circ}$ & -33.0 & -23.1 \\
\hline 5.0 & $1.08^{\prime}$ & $1.08^{\prime}$ & -37.2 & -37.2 & $0.96^{\prime}$ & $1.31^{\circ}$ & -33.0 & -23.1 \\
\hline
\end{tabular}

2 $\beta$ : Half-power beam width.

$\xi$ : First side lobe.

E: E plane.

$\mathrm{H}$ : H plane.

- The paraboloid focal ratio $F / d^{\prime}$ must be larger than 0.25 to avoid bad currents in the paraboloid and hence, the maximum zenith scan angle $\left|\psi_{\max }\right|$ of FAST can be only extended through to $69^{\circ}$ by an offset feed under the conditions of the geometry selected.

- When the distance $\delta_{\max }$ is limited to $0.70 \mathrm{~m}$ and the maximum zenith scan angles $\left|\psi_{\max }\right|$ are extended to $40^{\circ}, 45^{\circ}, 50^{\circ}, 55^{\circ}$ and $60^{\circ}$, the corresponding decrease in the antenna gain is by $1.2 \mathrm{~dB}, 2.0 \mathrm{~dB}, 3.0 \mathrm{~dB}, 4.2 \mathrm{~dB}$ and $5.7 \mathrm{~dB}$, respectively.

- The side lobes of the antenna pattern will increase with the increase of the zenith scan regions. When the maximum zenith scan angles $\left|\psi_{\max }\right|$ are extended from $30^{\circ}$ to $50^{\circ}$, the side lobes of the patterns at $\mathrm{H}$ plane increase from $-37.2 \mathrm{~dB}$ to $-23.1 \mathrm{~dB}$.

\section{References}

Kildal, Per-Sim, et al:: 1994, The Arecibo Upgrading: Electrical Design and Expected Performance of The Dual-Reflector Feed System, PIEEE 82(5) May, 714-724.

Milligan, Thomas A.: 1985, Modern Antenna Design, McGraw-Hill Book Company, New York.

Peng, Bo, et al: 1996, Proceedings Workshop on Large Antennas in Radio Astronomy, WWP 110, 151.

Qiu, Yuhai: 1998, A Novel Design for A Giant Arecibo-Type Spherical Radio Telescope With An Active Reflector, MNAS 301, 827-830. 